\title{
Social Recommendation Reseach for Building Optimization and Appropriate Social System using Individual Relationship Networks
}

\author{
S.P. Godlin Jasil, Pradeep Chand .N, M.Venkatesh
}

\begin{abstract}
Recommender frameworks are utilized to help clients in settling on decisions from different choices. Objective is to comprehend clients' inclinations and makes recommendations on suitable activities. A social recommender framework attempts to improve the exactness of traditional recommender frameworks by having the social trust between clients in interpersonal organizations into record. The Collaborative Filtering is utilized for the suggestion framework, to give the compelling recommendation to the individual client dependent on the surveys. The thing based is a type of coordinated effort framework dependent on the likeness between things determined utilizing individuals' evaluating of those things. The suggestion may contrast from client to client upon the information thickness for every client's thing rating and relationship system and it additionally develop after some time. The social recommender framework keeps up a controlled size of close/stable relationship organize for every client and endeavors to improve the exactness of regular recommender framework by taking the social intrigue and social trust between clients in informal community into record. . This examination proposes an way to deal with multifaceted nature of adding social connection systems to recommender frameworks. Our technique initially creates an individual relationship organize (IRN) for every client and thing by building up a novel fitting calculation of relationship systems to control the relationship spread and contracting. We at that point meld lattice factorization with social regularization and the area show utilizing IRN's to produce suggestions. Our methodology is very broad, and can likewise be connected to the thing relationship organize by exchanging the jobs of clients and things. Trials on different datasets with various sizes, levels of sparsity, and types of relationships demonstrate that our methodology can improve prescient precision and addition a superior versatility contrasted and best in class social recommendation strategies.
\end{abstract}

\section{INTRODUCTION}

As of late, online life has improved and now and then commanded many people's social exercises, interfacing them with their families, companions, partners, and even absolute outsiders. It has delivered rich social relationship data, for example, kinships in Facebook and Douban, adherents in Twitter, and trust in Epinions. The tremendous amount of online social connections not just makes it less demanding for people to impart their insights, anyway can likewise fill in as an extra cradle of data to updated basic rating. Recommender frameworks are used to help clients in settling on decisions from different options. They will likely comprehend clients' inclinations and makes

\footnotetext{
Revised Manuscript Received on July 18, 2019.

S.P. Godlin Jasil, Assistant professor, Dept. of CSE, Sathyabama University, Chennai, Tamilnadu, India.(email: spgodlinjasil@ gmail.com)

Pradeep chand .N, UG Student, Dept. of CSE, Sathyabama University, Chennai, Tamilnadu, India.

M.Venkatesh, UG Student, Dept. of CSE, Sathyabama University, Chennai, Tamilnadu, India.
}

recommendations on proper activities. A social recommender framework attempts to improve the precision of ordinary recommender frameworks by using the social trust between clients in informal organizations into record This methodology is to address the mixed unpredictability of social connections in social recommender frameworks. The methodology can be connected to both client and thing connections. It characterizes the individual relationship organize (IRN) for every client or thing, and present a calculation dependent on the similitude, thickness and certainty measures to make a harmony between its exactness and productivity.

The suggestion technique tends to the virus begin issue, catches the assorted variety of tastes between associated clients, and empowers adaptability by melding MF and also neighborhood models by means of IRN's. The strategy creates an individual relationship arrange for every client/thing to control the multifaceted nature of social relations. Additionally, existing social suggestion strategies endeavor to decrease information sparsity and cold-begin clients from the client perspective, however the cool begin issue for things still remains. This methodology consolidates thing relationship organize by utilizing client situated and thing focused point of view and can address the cool begin issue for things. The framework combine the area model and MF through IRN's to boost the capability of relationship arrange Dissimilar to the regularization technique with predefined similitudes in relationship organize, it demonstrates the taste decent variety between relationship individuals as unique closeness limitation to catch the timedeveloping nature of tastes in model learning. In contrast to prior works with a manual control of parity coefficient, our strategy focuses on the social impact as an additional client thing explicit inclination and retains the parity coefficient into an introduction weight lattice which speaks to the impact a client apply on another client, since the impact is found out from the information consequently.

Different models coordinating client thing rating framework and social relationship systems have been intended to give dynamic recommendation and to ease the absence of data.

Present social recommenders utilize the area strategies [15] or network dividing (MF) methods [16], [17], [18] as base models. Regardless of developing acknowledgment in true applications, a few difficulties still cutoff the precision and proficiency of social recommender frameworks because of the accompanying attributes of social connections [24, 


\section{SOCIAL RECOMMENDATION RESEACH FOR BUILDING OPTIMIZATION AND APPROPRIATE SOCIAL SYSTEM USING INDIVIDUAL RELATIONSHIP NETWORKS}

25]. As a matter of first importance, most existing MFbased social recommendation techniques accept that a major enough relationship organize is accessible for every client to address the information sparsity and the (new client) coldbegin issues. In any case, with the quick increment on the quantity of clients on Internet, numerous clients may fabricate associations with just a couple among the a huge number of clients [19], [20]. The total client relationship arrange is colossally extensive, yet inadequate and unequal. Some dynamic clients have relations with other dynamic clients that have given numerous item evaluations. However, clients with inadequate rating information themselves may likewise have only a couple of client associations. Subsequently, the cool begin issue could turn out to be more terrible. Given the meager and lopsided rating lattice, the commitment of relationship systems to a recommender model may contrast from client to client contingent upon the information densities of every client's thing evaluations and relationship arrange and furthermore develop after some time. Social recommender frameworks utilizing accessible connections may pick up a little or even no improvement contrasted with conventional recommender frameworks [3].

Second, a general presumption compared to social suggestion techniques is the inclination of a client is like and can be affected by his/her social relationship organize [1]. The speculation may not generally be valid since the flavors of one's companions may fluctuate fundamentally. Because of the minimal effort of framing on the web associations nowadays, associated clients are not really such comparative. In this way, relations of social are blended with helpful and loud associations that can really acquaint bad data with recommender frameworks [22]. Hence social recommender frameworks should treat social connection individuals diversely dependent on how comparable they are. At last, the multifaceted nature for finding the closest neighbors (comparative or confided in clients) in a huge, unequal and loud social relationship arrange is restrictively high. Showing expectations to numerous online clients in a constrained time is turning into a noteworthy test for online administrations. Social recommender frameworks utilizing the total relationship arrange in their models may bring clients late online encounters. Hence social recommender frameworks should keep up a controlled size of close/stable relationship arrange for every client.

\section{RELATED WORK}

Memory Based Methods. Memory-based strategies produce expectation utilizing the entire client thing rating framework or a few examples. The techniques can be additionally isolated into client arranged strategies [15] and thing focused strategies. The two methodologies depend on the area models which are the most widely recognized techniques for CF. Neighborhood models are fixated on discovering connections between clients or, on the other hand, things. A client situated methodology assesses the inclination of a client to a thing dependent on appraisals of comparable clients on a similar thing. A thing focused methodology assesses the inclination of a client for a thing dependent on his/her appraisals of "neighboring" things. Explicit calculations change by picking diverse similitude measures, for example, Pearson connection, vector cosine, Jaccard, and mean supreme contrast . One might say, these techniques change the client thing space by review them as gatherings of likeminded clients or comparative things. As the quantity of clients and things builds, neighborhood strategies experience the ill effects of the computational multifaceted nature of the closest neighbors look in highdimensional spaces.

Demonstrate Based Methods. Show based strategies utilize a model to create evaluations and apply information mining and AI procedures to discover designs from the preparation information, which can also be utilized to do forecasts for obscure. Contrasted and memory-based CF, show based CF has an increasingly comprehensive objective to reveal idle factors that clarify watched evaluations [3]. Inert factor models, for example, PLSA, neural systems, idle dirichlet portion [13], and particular esteem deterioration (SVD), involve an elective methodology by changing the two things and clients to the equivalent idle factor space. Probably the best acknowledge of idle factor models depend on grid division. MF-based CF models [16], [17], [18] accept that a couple of inactive examples impact client rating practices and play out a low-position network factorization on the client thing rating lattice to viably manage substantial datasets. This regularly raises troubles inferable from the high segment of missing qualities brought about by meager condition in the client thing rating network. Besides, the framework learns/trains the model by fitting recently watched appraisals and requirements to maintain a strategic distance from over fitting the watched information by regularizing the educated parameters. In this way, the primary disadvantage of this learning technique for MF is the manual multifaceted nature control to produce a fitting model, especially in scanty and uneven datasets.

Display based CF approaches as a rule have a superior versatility yet a lower exactness, contrasted and memory contained $\mathrm{CF}$ approaches which have the superior precision yet a lesser adaptability. Conventional recommender frameworks expect that clients are autonomous and indistinguishably conveyed. Social recommendation use client connections to improve the execution of suggestion dependent on the instinct about social impact and the rule of homophily. Most existing social recommender frameworks pick CF models as their fundamental models to manufacture frameworks. A CF-based social recommendation technique can likewise be arranged into memory-based and show based strategies [2], [3].

Memory Based methods. Two key issues for a memory based CF technique are processing the closeness to discover neighbors and totaling evaluations to create forecasts. The relationship systems can be connected in memory-based CF techniques since informal communities give proof to comparability. Clients with closer social connections to others are bound to be trusted and are all the more dominant on affecting others. Many existing methodologies for social suggestion are neighborhood models, for example, Tidaltrust, Moltrust, Advogato, AppleSeed , and Trust- 
Walker [8]. These methodologies misuse different complex calculations to process an area of confided in clients in informal communities who have evaluated the objective thing. They at that point total confided in clients' evaluations, weighted by the trust esteems, to process the correct rating expectation. Tidal trust plays out a changed broadness inquiry in interpersonal organizations to figure a forecast. Advogato utilizes a most extreme stream based way to deal with discover the area in rating expectation. The fundamental instinct of AppleSeed is roused by spreading the actuation display. TrustWalker plays out a few arbitrary strolls on the interpersonal organization. Neighborhood strategies depending on a couple of noteworthy neighborhood relations are best at identifying much restricted connections however can't catch the totality of feeble signs enveloped in every one of the evaluations of a client or a thing.

Show Based Methods. Demonstrate based social recommender frameworks pick display based $\mathrm{CF}$ techniques as their essential models. Most existing social recommender frameworks in this classification use grid factorization to learn inactive variables for clients and things from coordinating the client thing rating lattice and the informal community. Mama et al. [5] propose a probabilistic factor examination system called social suggestion (SoRec). SoRec performs co-factorization in the client thing network and the client

social connection framework by having a similar client inclination dormant factor. Furthermore, propose a comparable model. The favorable position of factor examination approaches is they perform recommendation and social connection expectation together. In their subsequent work, Ma et al. [6], utilize the expression "social trust gathering" (RSTE) to speak to the definition of social trust confinements on recommender frameworks. What's more, Yeung and Iwata likewise fuse the current evaluations from interpersonal organizations to anticipate rating. A missing rating for a given client is anticipated by a direct blend of appraisals from the client and his/her interpersonal organization. The group techniques include physical translations of suggestion, i.e., a client's last evaluating choice is the harmony between this present client's very own taste and his/her confided in clients' favors, contrasted and the factor investigation strategy. Anyway one fundamental downside of the gathering techniques is the manual control of the equalization. Guo et al. [14] propose a SVD++ [17] based TrustSVD display which joins the element of both cofactorization and group techniques to accomplish a superior precision.

Jamali and Ester [9] join trust spread in informal community into lattice factorization (SocialMF) to make the inclination of a client near the normal inclination of his/her interpersonal organization. SocialMF makes the highlights of each client reliant on highlight vectors of companions of companions in an interpersonal organization. Mama et al. [7], characterize "social regularization" (SR) to speak to the social requirements on recommender frameworks. Regularization strategies accept that a client inclination ought to be like that of his informal organization. The inclination closeness of two associated clients is constrained by their likenesses dependent on their past evaluations.
Favorable position of regularization approaches is that they in a roundabout way demonstrate the proliferation of inclinations in informal communities, which can decrease cold-begin clients and increment the inclusion of things suggested [3]. In the recent years, Cloud Computing has emerged as an alternate to the acquisition and management of resources [21].

\section{EXISTING SYSTEM}

In existing social recommender framework incorporated distinctive models, for example, neighborhoods strategy and lattice factorization. In spite of developing acknowledgment in true applications, a few difficulties still point of confinement the exactness and productivity of social recommender frameworks because of the accompanying qualities of social connections. most existing MF-based social recommendation strategies accept that a major enough relationship organize is accessible for every client to address the information sparsity and the (new client) cold-begin issues. Be that as it may, with the fast increment on the quantity of clients on Internet, numerous clients may fabricate associations with just a couple among the a large number of clients. The total client relationship organize is massively huge, yet inadequate and lopsided. Some dynamic clients have relations with other dynamic clients that have given numerous item evaluations. In any case, clients with lacking rating information themselves may likewise have only a couple of client associations. Therefore, the cool begin issue could turn out to be more awful. Given the inadequate and unequal rating framework, the commitment of relationship systems to a recommender model may contrast from client to client contingent upon the information densities of every client's thing appraisals and relationship arrange and furthermore develop after some time.

\subsection{DISADVANTAGES OF THE EXISTING SYSTEM}

- $\quad$ Less Efficiency. 


\section{PROPOSD SYSTEM}

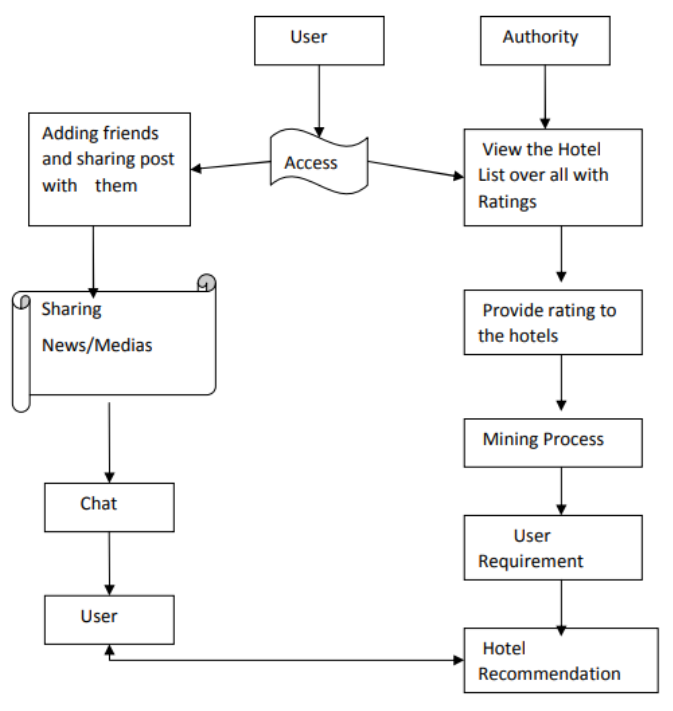

We propose the best way to deal with location the mixed multifaceted nature of social connections in social recommender frameworks. The methodology can be connected to both client and thing connections. We characterize the individual relationship arrange (IRN) for every client or thing, and present a calculation dependent on the comparability, thickness and certainty measures to make a harmony between its exactness and productivity. The suggestion technique tends to the virus begin issue, catches the assorted variety of tastes between associated clients, and empowers adaptability by combining MF and neighborhood models by means of IRN's. Our strategy produces an individual relationship arrange for every client/thing to control the multifaceted nature of social relations. In addition, existing social suggestion techniques attempt to diminish information sparsity and cold-begin clients from the client point of view, yet the chilly begin issue for things still remains. Our methodology consolidates thing relationship organize by utilizing client arranged and thing focused point of view and can address the chilly begin issue for things.

We intertwine the area model and MF by means of IRN's to boost the capability of relationship arrange. Not at all like the regularization strategy with predefined similitudes in relationship arrange, our technique models the taste decent variety between relationship individuals as powerful comparability requirement to catch the time-developing nature of tastes in model learning. In contrast to prior works with a manual control of parity coefficient, our technique focuses on the social impact as an additional client thing explicit predisposition and ingests the parity coefficient into an introduction weight lattice which speaks to the impact a client apply on another client, since the impact is found out from the information naturally.

\subsection{ADVANTAGES OF THE PROPOSED SYSTEM}

- Improves Efficiency.

- Improves Accuracy.
Fig 1 :Overview Of The Proposed System

\section{SYSTEM MODEL \& RESULTS}

\subsection{SOCIAL NETWORKING APPLICATION}

In this module, the OSN web application is work as long range interpersonal communication application in which new client can enroll for the administrations. The enrollment fields are approved client can login with his qualifications. The clients can set Cover picture, Profile photographs and can include companions. The companion demand will be dispatched to end client account and will be promptly accessible once he signed in. He can acknowledge/dismiss the companion ask. The companion list is appeared in the correct board and can almost certainly visit with the beneficiary private way.

\subsection{DATA SHARING WITH ACCESS CONTROL}

In this Module, The clients can post some News, Images and Video and some other data. These posts can be imparted to companions with access control. The common posts can be seen by companions on the off chance that they have legitimate access control once they login. Companions can answer to the posts with a few remarks and like/loathe the posts.

\subsection{USER VIEW AND RATING SYSTEM}

In this Module, to gather the client companions list from interpersonal organization. Furthermore, client sees the all inn names and its general appraisals. To look through the predefined lodging rating data in the database. The client can be evaluated to the chose inn and its properties (e.g.: rating generally speaking, rating room, rating neatness, etc). The lodging rating credits are refreshed to the database.

\subsection{SOCIAL RECOMMENDER SYSTEM}

In this Module, The mining procedure is performed to sift the inn list.to channel through the companions and companions of companions appraised lodging rundown and show them to the client. The client gives the prerequisite for lodging (e.g.: tidiness, room, administration, Location, and so on). The clients' inclinations are cumulated dependent on client necessity. The client necessities are find to the companions list, the required rundown not in the companions appraised rundown to discover the companion of companions evaluated rundown. At last prescribe the client necessity inn.

\section{CONCLUSION}

This paper presents asocial suggestion approach that abuses singular relationship systems (IRN's) for clients. What's more, things to address the gigantic size, sparsity, awkwardness and commotion in relationship systems and to improve productivity and precision of social recommender framework. This suggestion approach improves the exactness by adaptively taking care of the exchange off between individual inclinations and encounters and social impact, considering the decent variety of tastes between relationship individuals. This strategy further empowers the adaptability for relationship organizes by sifting through 
commotion and excess associations of relationship arranges in the meantime. An exploratory examination on four datasets from Epinions, Flixster, and Doubanand Netflix has been led. The outcomes demonstrate that the proposed methodology accomplishes a superior expectation precision and versatility as a rule. In addition, the outcomes demonstrate that utilizing IRN's in thing suggestion improves the adaptability without losing precision much of the time. The outcomes likewise demonstrate that every social relationship ought not be viewed as equivalent in social recommender systems[24]. The present investigation endeavors to all eviate the intrinsic issues of the social recommender frameworks and match the necessities of suggestion exactness and versatility. Nonetheless, execution improvement is as yet feasible for future work. To start with, this investigation features the significance of the informational collection with relationship systems. In the event that the crossing point of the busi-ness with client data space is meddling or addsclutter, endeavors can fizzle and may empty an incentive out of clients and online networks. Consequently, safeguarding protection while utilizing informal organizations ought to be considered. This examination likewise centers around mining the connections among clients and between things however has low thought for the setting of clients and things (e.g., time, area, feeling and dynamic status)[48].Many application situations depend just on the connections among clients or potentially things that don't create successful recommendation. The proposed methodology is general and can be effectively reached out to consolidate other logical data.

\section{REFERENCES}

1. X. Yang, H. Steck, and Y. Liu, "Circle-based recommendation in online social networks," in Proc. 18th ACM SIGKDD Int. Conf. Knowl. Discovery Data Mining, 2012, pp. 1267-1275.

2. X. Yang, Y. Guo, Y. Liu, and H. Steck, "A survey of collaborative filtering based social recommender systems," Comput.Commun., vol. 41, pp. 1-10, 2014.

3. J. Tang, X. Hu, and H. Liu, "Social recommendation: A review," Social Netw. Anal.Mining, vol. 3, no. 4, pp. 1113-1133, 2013.

4. Y.-M. Li, C.-T.Wu, and C.-Y. Lai, "A social recommender mechanism for e-commerce: Combining similarity, trust, and relationship," Decision Support Syst., vol. 55, no. 3, pp. 740-752, 2013.

5. H. Ma, H. Yang, M. R. Lyu, and I. King, "SoRec: Social recommendation using probabilistic matrix factorization," in Proc.17th ACM Conf. Inf. Knowl. Manage., 2008, pp. 931-940.

6. H. Ma, I. King, and M. R. Lyu, "Learning to recommend with social trust ensemble," in Proc. 32nd Int. ACM SIGIR Conf. Res. Develop. Inf. Retrieval, 2009, pp. 203 210.

7. H. Ma, D. Zhou, C. Liu, M. R. Lyu, and I. King, "Recommender systems with social regularization," in Proc. 4th ACM Int. Conf. Web Search Data Mining, 2011, pp. 287-296.

8. M. Jamali and M. Ester, "TrustWalker: A random walk model for combining trust-based and item-based recommendation," in Proc. 15th ACM SIGKDD Int. Conf. Knowl. Discovery Data Mining, 2009, pp. 397406.

9. M. Jamali and M. Ester, "A matrix factorization technique with trust propagation for recommendation in social networks," in Proc. 4th ACM Conf. Recommender Syst., 2010, pp. 135-142.

10. Q. Yuan, L. Chen, and S. Zhao, "Factorization versus regularization: Fusing heterogeneous social relationships in top-N recommendation," in Proc. 5th ACMConf. Recommender Syst., 2011, pp. 245-252.

11. J. Noel, et al., "New objective functions for social collaborative filtering," in Proc. 21st Int. Conf. World Wide Web, 2012, pp. 859-868.

12. C. C. Chen, Y.-H.Wan, M.-C.Chung, and Y.-C. Sun, "An effective recommendation method for cold start new users using trust and distrust networks," Inf. Sci., vol. 224, pp. 19-36, 2013.

13. C. Chen, X. Zheng, Y. Wang, F. Hong, and Z. Lin, "Context-aware collaborative topic regression with social matrix factorization for recommender systems," in Proc. 28th AAAI Conf. Artif. Intell., 2014, pp. 9-15.

14. G. Guo, J. Zhang, and N. Yorke-Smith, "TrustSVD Collaborative filtering with both the explicit and implicit influence of user trust and of item ratings," in Proc. 29th AAAI Conf. Artif. Intell., 2015, pp. 123-129.

15. H. Liu, Z. Hu, A. Mian, H. Tian, and X. Zhu, "A new user similarity model to improve the accuracy of collaborative filtering," Knowl.-Based Syst., vol. 56, pp. 156-166, 2014.

16. A.Mnih and R. Salakhutdinov, "Probabilistic matrix factorization," in Proc. Advances Neural Inf. Process. Syst., 2007, pp. 1257-1264.

17. Y. Koren, R. Bell, and C. Volinsky, "Matrix factorization techniques for recommender systems," Comput., vol. 42, no. 8, pp. 30- 37, 2009.

A. K. Menon and C. Elkan, "A log-linear model with latent features for dyadic prediction," in Proc. 10th Int. Conf. Data Mining, 2010, pp. 364-373.

18. J. Xin, Z. Wang, L. Qu, and G. Wang, "Elastic extreme learning machine for big data classification," Neuro computing, vol. 149, pp. 464-471, 2015.

19. S.-R. Yan, X.-L.Zheng, Y. Wang, W. W. Song, and W.Y. Zhang, "A graph-based comprehensive reputation model: Exploiting the social context of opinions to enhance trust in social commerce," Inf. Sci., vol. 318, pp. 51-72, 2015.

20. S.P. Godlin Jasil , M. Deepa(2014). Efficient Utilization of Cloud Infrastructure by Handling Heterogeneous Workloads. International Journal of Applied Engineering Research. Volume 9, Number 23 (2014) pp. 1365513666.

21. Sethuraman, R., Krishna Chaitanya Reddy, V., Gautham Veer, M., Subhashini, R., "Identifying trends in facebook usage: A visual approach", International Journal of Recent Technology and Engineering, 2019.

22. P. Ajitha, Dr. G. Gunasekaran.," Effective Feature Extraction For Document Clustering To Enhance Search Engine Using Xml", Journal of Theoretical and Applied Information Technology, Little Lion Scientific ,PP 2026,10th October 2014. Vol. 68 No.1. EISSN 18173195 ISSN 19928645.

23. T.R.Sowmiya,S.Revathy, Survey on Big Data Threats in Online Social Media, Proceedings of the 2018 IEEE International Conference on Communication and Signal Processing, ICCSP 2018.

24. Vijeya Kaveri, V.,Maheswari, V, A model based resource recommender system on social tagging data, International Journal of Science and Technology, Vol 9(25), July 2016 